\title{
Recurrent cerebral vasospasm after aneurysmal subarachnoid hemorrhage- A temporal "Marching Pattern" of progression
}

Keywords: cerebral vasospasm, Marching cerebral vasospasm, angiographic pattern

Abbreviations: CVS, cerebral vasospasm; aSAH, aneurysmal subarachnoid hemorrhage; CT, computed tomograph; ICA, internal carotid artery; MCA, middle cerebral artery; M1, proximal segment of middle cerebral artery; A1, proximal segment of anterior cerebral artery; M2, M3, distal segments of middle cerebral artery; A2, A3, distal segments of anterior cerebral artery; IA, intraarterial

\section{Introduction}

Cerebral vasospasm (CVS) following aneurysmal SAH is an important cause of morbidity and mortality and its pathogenetic mechanisms remain controversial. The available literature on CVS describes the angiographic patterns of evolution/resolution over time and the diffuse /focal nature of involvement of the cerebral vessels. However, the temporal pattern of evolution and mode of spread along the arterial tree in a given patient has been infrequently described.

We report a patient of aneurysmal SAH in the post-clipped status who developed recurrent episodes of vasospasm over a period of 2 weeks requiring repeated intraarterial spasmolysis. Temporally the CVS involved a new distal portion of the arterial tree each time spreading in a spasmodic squeezing pattern along the vessel length, a phenomenon we named as "Marching cerebral vasospasm."

We discuss its possible pathogenetic mechanisms in light of available literature.

\section{Case report}

A 43-year old female without any relevant medical history was hospitalized after severe headache, neck pain, and self-limiting cramp of both upper extremities. At admission, she was awake and oriented without any focal neurological deficits. Brain computed tomography (CT) showed diffuse subarachnoid hemorrhage in all cisterns without intracerebral or intraventricular hemorrhage (Fisher grade 3, BNI grade 4) nor accompanying hydrocephalus. The CT angiography showed an anterior communicating artery aneurysm which was clipped six hours after the initial hemorrhage without any complications. A cisternal drain was left behind to drain cerebrospinal fluid. This drain was removed four days after surgery. Postoperative she was observed in our neurointensive care unit according to standard protocol. Due to the anatomy of the cranial vault, no transcranial Doppler could be performed.

At post hemorrhage day seven, the patient suddenly developed neurological deficits, including a decreased Glasgow coma score (GCS) E4M5V2, global aphasia and, a diffuse paresis of the right arm with normal leg function. She was diagnosed to have suffered symptomatic vasospasm. After a CT brain to rule out major infarcts
Volume 10 Issue 6 - 2020

\author{
Ahmed AR Maqsood,' Andaka Deddy,2 \\ Balakrishnan Shankar, ${ }^{3}$ Talla Bhushanam \\ Vinay, ${ }^{4}$ Germans Menno, ${ }^{5}$ Keller Emanuela, ${ }^{5}$ \\ Hakim Husain Shakir ${ }^{6}$ \\ 'Department of neurosurgery,Al Ansaar and Columbia Asia \\ Hospitals, Mysuru, India \\ ${ }^{2}$ Department of Neurology Interventional, Siloam hospital Bali, \\ Indonesia \\ ${ }^{3}$ Department of Neurology, MIOT Hospitals, India \\ ${ }^{4}$ Department of Neurosurgery, PINNACLE hospital \\ vishakapatnam, India \\ ${ }^{5}$ Universitäts Spital Zürich Klinik für Neurochirurgie, \\ Frauenklinikstrasse 10 809। Zurich, Switzerland \\ ${ }^{6}$ Department of Neuroradiology University Hospital Zurich, \\ Frauenklinikstrasse $10 \mathrm{CH}-809$ I Zurich, Switzerland
}
Correspondence: Hakim Husain Shakir, Department of Neuroradiology University Hospital Zurich, Frauenklinikstrasse $10 \mathrm{CH}-809$ | Zurich, Switzerland, Tel ++4 I-44-255 5600 , Email shakir.husain@usz.ch

Received: August 12, 2020 | Published: December 18, 2020

a diagnostic cerebral angiogram was done along with intraarterial vasospasmolysis with nimodipine. After initial improvement, she again developed the same focal deficits, with now also a leg paresis, within twelve hours after spasmolysis which prompted us to perform a second emergency spasmolysis. She was extubated the next day and neurological examination showed global aphasia with improved right-sided hemiparesis. Some hours later she developed a status epilepticus for which she needed reintubation and sedation. Invasive multimodality monitoring (ICP, PtiO2, and microdialysis) was placed right frontal. The $\mathrm{PtiO} 2$ and microdialysis showed evidence for progressive functional deterioration and after interdiciplinary discussion, we decided to perform a third and even fourth spasmolysis over the next 4 days. These successive treatments lead to improved multimodality parameters.

The serial angiographic images are depicted in figures 1 to 4 . We can appreciate that over a period of 6 days in the anterior circulation the CVS has progressed from terminal ICA, and M1M2 segments and spreading distally over the arterial tree to involve the M3 M4, A3 A4 segments, and distal PCAs. Each time after lysis the spasm got relieved and when it recurred it did not appear in a segment in which it existed but was more pronounced distally in a new segment of the same vessel as if the vessel is getting squeezed distally. We call this phenomenon a "Marching Pattern". 

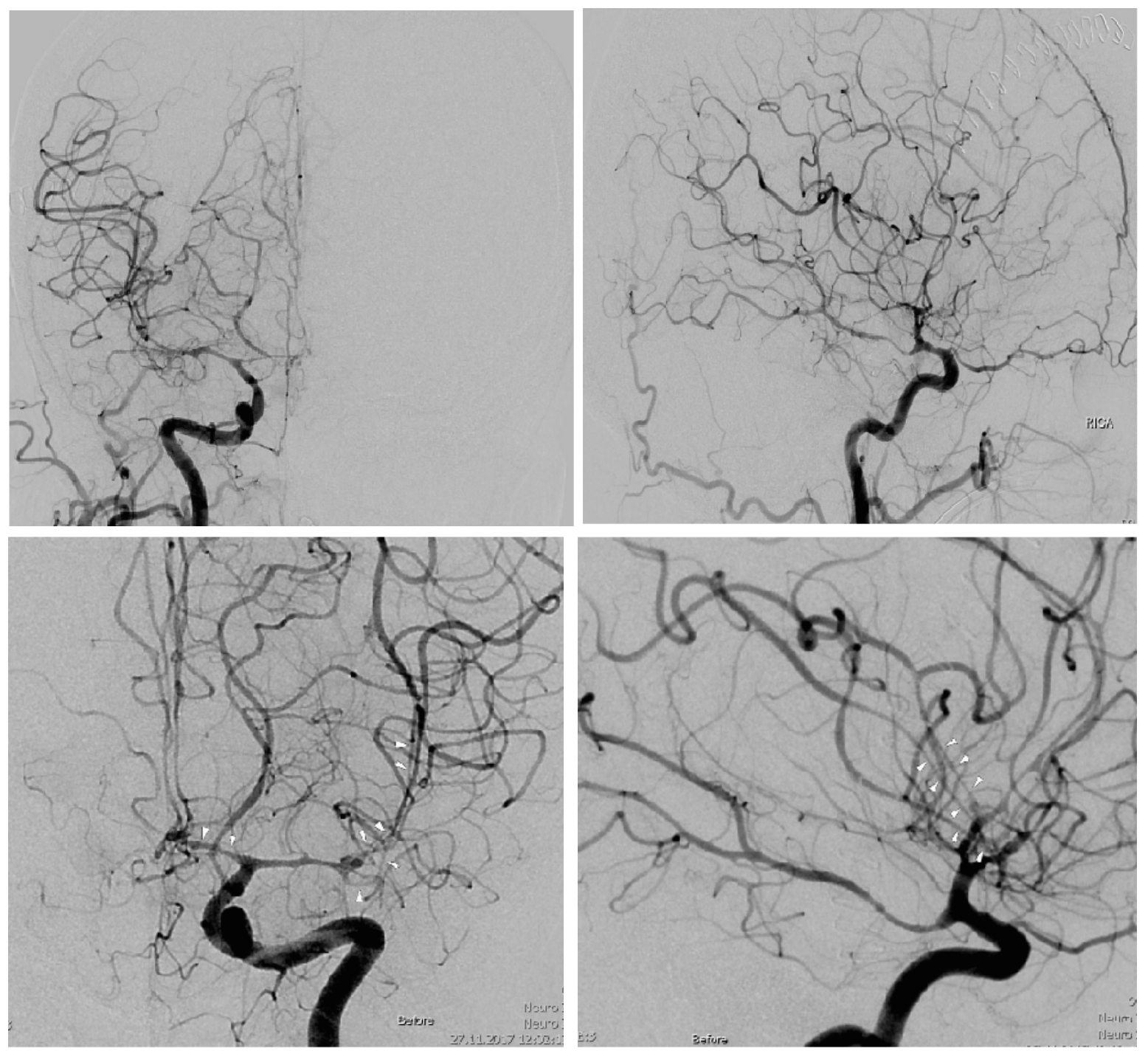

Figure I I ${ }^{\text {st }}$ episode of CVS. Distal ICA,AI, MI, M2 and proximal PCOM in spasm, both sides.
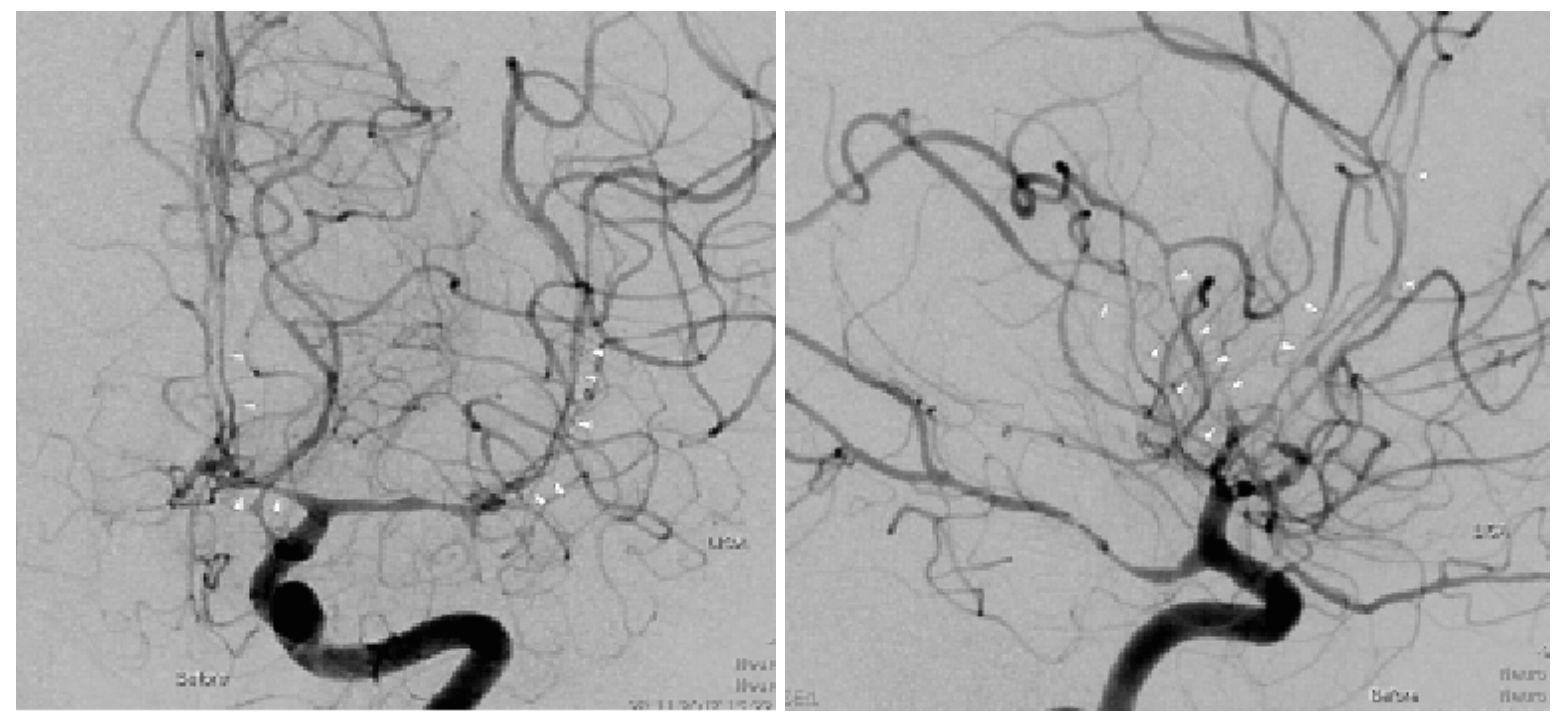

Figure $22^{\text {nd }}$ episode of CVS-distal ICA snd proximal PCOM are normal, CVS involves MIAI. 

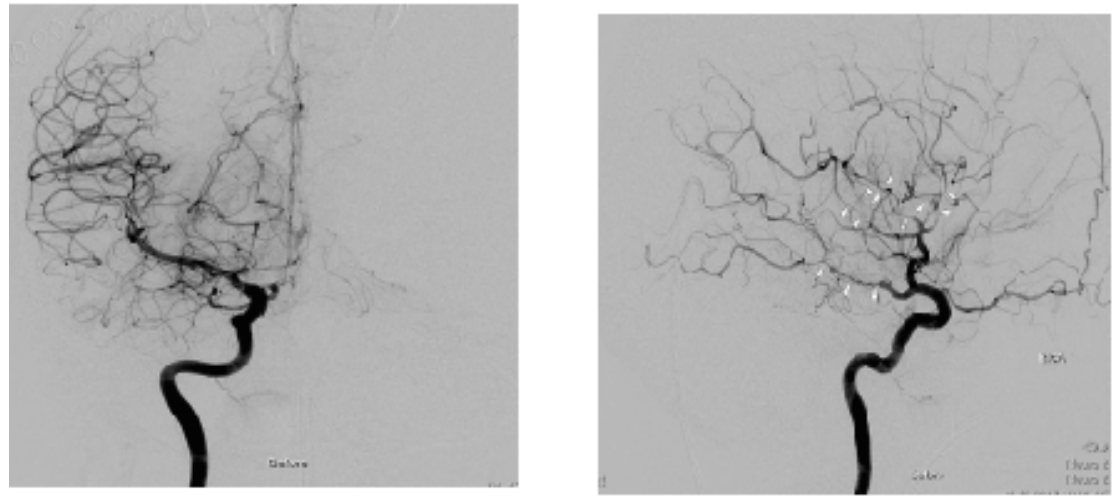

\section{RICA}
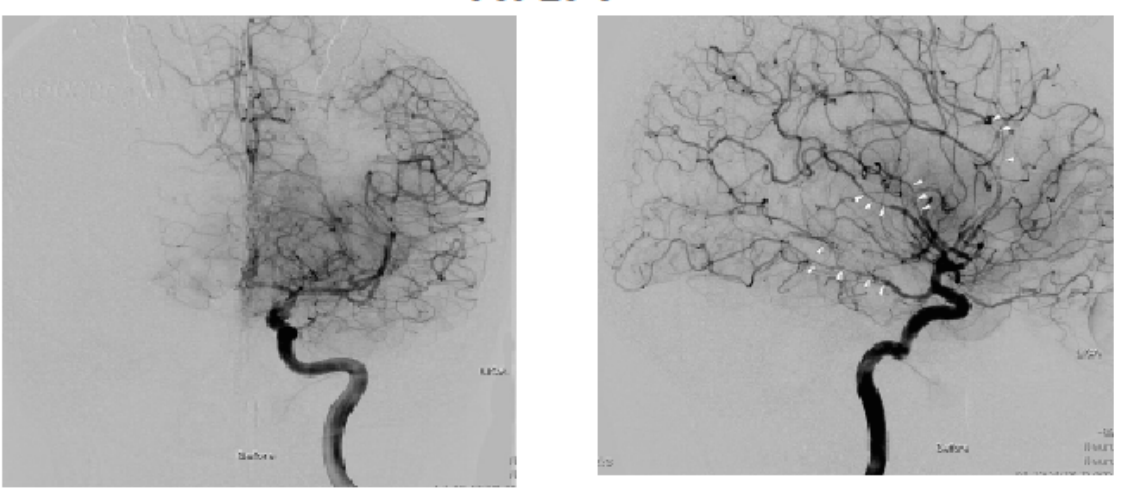

\section{LICA}

Figure $33^{\text {rd }}$ episode of CVS. Proximal ICA, MI looks normal. CVS seen in distal M2M3.
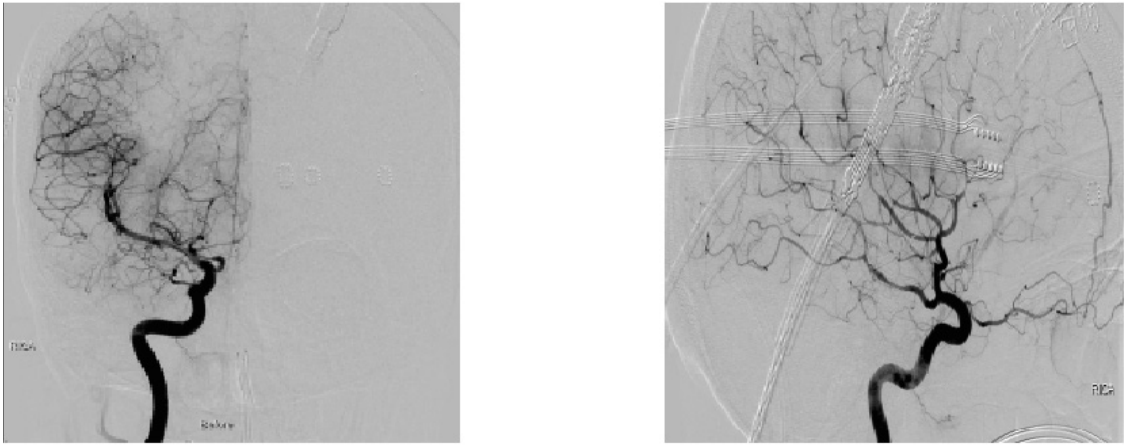

\section{RICA}
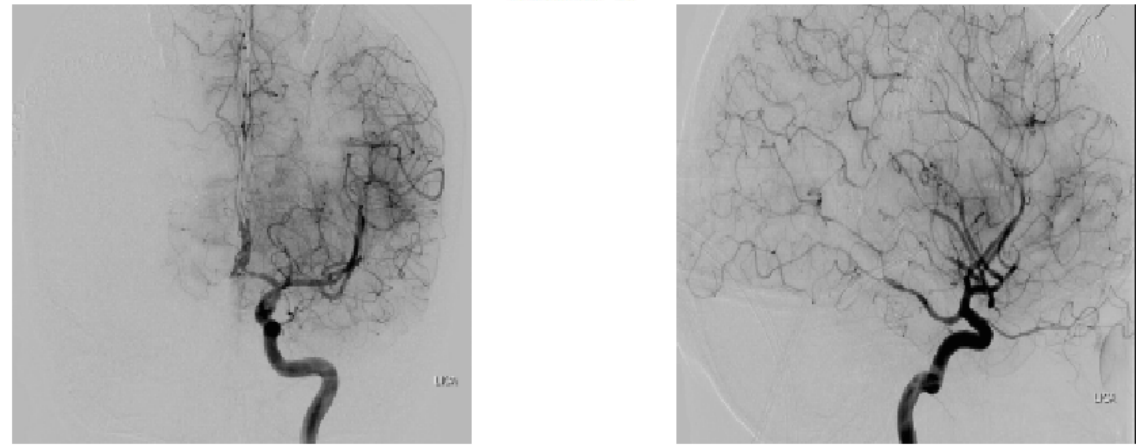

\section{LICA}

Figure $44^{\text {th }}$ episode of CVS. Involves the M3, M4 and A3, A4 segments.

Citation: Maqsood AAR, Deddy A, Shankar B, et al. Recurrent cerebral vasospasm after aneurysmal subarachnoid hemorrhage- A temporal "Marching Pattern" of progression.J Neurol Stroke. 2020;10(6):2 I8-222. DOI: 10.15406/jnsk.2020.10.00443 


\section{Discussion}

CVS following aneurysmal subarachnoid hemorrhage (aSAH), is an important cause of significant morbidity and mortality. ${ }^{1}$ It is consistently the leading cause of poor outcome and death, adversely affecting more than one in five of all patients who have suffered SAH. ${ }^{2}$

Worthwhile improvements in the available treatments have been made during the last 3 decades, resulting in a definite reduction of morbidity and mortality attributed to it from $25-30 \%$ in the 1970 s to $15-20 \%$ in the 1980 s, to $5-10 \%$ currently. ${ }^{3}$ Nonetheless, there remains an absence of consistently efficacious and ubiquitously applied preventive and therapeutic interventions for this cause of significant mortality and morbidity, even now. This probably reflects the fact that despite ongoing research efforts the underlying pathogenic mechanisms remain inconclusive. ${ }^{4}$

The angiographic descriptions of vasospasm after SAH and its progression/regression over time have been described as early as $1951 .{ }^{5}$ Various studies document the onset and resolution with serial angiograms. Vasospasm has its onset in man about Day 3 after subarachnoid hemorrhage, is maximal at Days 6 to 8 , and is gone by Day 12.

Sano et all described types of CVS and divided them into Type 1- extensive diffuse, Type 2 Multi segmental, and Type 3 Focal and found that surgical intervention in Type 1 had the worst results. ${ }^{7}$ In most of the above studies, onset and severity of spasm over a period of time were discussed but none documented that in a given patient there could be a defined pattern of how the vasospasm would spread over the intracranial arterial tree.

Jones et described patterns of evolution of CVS temporally in a cohort of coiled and clipped patients. ${ }^{8}$ In their study they found perianeurysmal spasm affected all subjects. However, whereas spasm remained largely confined in the clipped group, patients who underwent coiling developed stepwise progression distally over time. In one of their illustrative cases, spasms involving bilateral A1, A2, M1, and M2 segments on post-bleed day 5 moves distally by day 7 to the posterior circulation; the right M1 and M2 segments had improved by then. By post-bleed day 15 , much of the anterior circulation spasm had improved whereas the basilar and posterior cerebral arteries appear more affected. Their report does not talk of spasm spreading distally on the same-named vessels.

Contrary to this in our patient, the CVS was recurrent despite intraarterial spasmolysis, and it demonstrated downstream spread into the same vascular territory but with the release of the spasm in the proximal portion of the same vessel in a squeezing fashion and that is why we call this a Marching Pattern. In one of the widely accepted theories of vasospasm, the volume of subarachnoid blood on presentation is an important predictor of angiographic vasospasm. The clot size and density and its clearance rate are important in determining the risk of vasospasm and delayed ischemic neuro deficits after the SAH. ${ }^{9}$

Subarachnoid hematoma evacuation during surgery was initially reported to reduce the incidence of vasospasm. ${ }^{10}$ However, further studies have shown that the method of aneurysm treatment does not affect clot clearance after aneurysmal subarachnoid hemorrhage. ${ }^{11}$

In a review article on the pathogenesis of vasospasm Kolias et al have shown that after aneurysmal SAH, a multifaceted cascade of events is initiated, ultimately leading to vasospasm. ${ }^{12}$ Breakdown products of blood in the subarachnoid space probably are the triggering factor, while calcium-dependent and independent vasoconstriction is taking place during CVS. Lipid peroxides, an imbalance between endothelium-derived vasoconstrictor and vasodilator substances, nitric oxide toxicity, arachidonic acid metabolites, inflammatory cascades, disruption of neuronal mechanisms that regulate vascular tone, endothelial proliferation, and apoptosis, are among those factors that, acting through interconnected pathways, result in the development of vasospasm.

In yet another study it was shown that endothelial glycocalyx injury occurs in $\mathrm{SAH}$, and might contribute to delayed cerebral ischemia by regulating cerebral micro thrombosis and delayed neuroinflammation. ${ }^{13}$ In an article titled "anatomical observations of the subarachnoid cisterns of the brain during surgery" Yasargil et all have observed that although often considered as anatomically distinct compartments, the basal cisterns are, in fact, only separated from each other by a trabeculated porous wall with various sized openings. ${ }^{14}$ These apertures can become plugged and partially or obliterated after subarachnoid hemorrhage and in cases of basal tumor. The arachnoid fibers and membranes are noted to be regularly thicker and tougher where the arteries pass through the trabeculated wall from one cisternal compartment to another.

Neurogenic factors, although not thought to be important in the normal physiological regulation of cerebral arteries, may become important under pathological conditions such as acute SAH. The presence of a rich plexus of adrenergic fibers within the adventitial layer of the pial vasculature has been amply documented. Further, there is evidence of differential innervation of nerve endings along the arterial tree and also between the anterior and posterior circulation. Also, the level of circulating catecholamines usually increases after SAH. It is possible, then, that this increase in circulating catecholamines, associated with abnormal sensitivity of the cerebral vessels to catecholamines and a differential innervation, is a factor in the complex genesis and progression of vasospasm. ${ }^{15,16}$ There is also evidence of sphenopalatine ganglion stimulation decreasing vasospasm and increasing cerebral blood flow after SAH in monkeys. This was associated with the opening of the blood-brain barrier. ${ }^{17}$ This also signifies the role of neurogenic mechanisms.

\section{Conclusion}

In light of the above literature review, we hypothesize that due to thickened arachnoidal bands near major vessels the SAH remained confined near proximal arteries initially and later the blood spread through opened cisterns (due to surgery) and also through naturally existing pores between cisterns. The vasospasm also followed the spread of the blood with its toxic metabolites as mentioned above and was influenced in some way by the neural innervations. Hence the "Marching pattern."

\section{Acknowledgments}

None.

\section{Conflicts of interest}

The authors declare no conflict of interest.

\section{References}

1. Kassell NF, Torner JC, Haley EC Jr, et al. The International Cooperative Study on the timing of aneurysm surgery. Part 1: Overall management results. J Neurosurg. 1990;73(1):18-36.

2. Dorsch NW. Cerebral arterial spasm - a clinical review. Br J Neuro Surg. 1995;9(3):403-412. 
3. Grieve J, Kitchen N. Aneurysmal subarachnoid hemorrhage. In: Moore A, Newell D, editors. Neurosurgery: principles and practice, London: Springer-Verlag Ltd. 2003 p 315-332.

4. Dumont AS, Dumont RJ, Chow MM, et al. Cerebral vasospasm afte subarachnoid hemorrhage: putative role of inflammation. Neurosurgery. 2003;53(1):123-133.

5. Arthur Ecker, AD Paul A. Riemenschneider M.D. Arteriographic demonstration of spasm of the intracranial arteries with special reference to saccular aneurysms. J Neurosurg. 1951;8(6):660.

6. Bryce Weir, Michael Grace, John Hansen, et al. Time course of vasospasm in man. J Neurosurg. 1978;48:173-178.

7. K Sano, I Saito. Timing and Indication of Surgery for Ruptured Intracranial Aneurysms With Regard to Cerebral Vasospasm. Acta Neurochirurgica. 1978;41(1-3):49-60.

8. J Jones, J Sayre, R Chang, et al. Cerebral vasospasm patterns following aneurysmal subarachnoid hemorrhage: an angiographic study comparing coils with clips. J Neuro Intervent Surg. 2014;7(11):803-807.

9. Reilly C, Amidei C, Tolentino J, et al. Clot volume and clearance rate as independent predictors of vasospasm after aneurysmal subarachnoid hemorrhage. J Neurosurg. 2004;101(2):255-261.

10. Mizukami M, Kawase T, Usami T, et al. Prevention of vasospasm by early operation with removal of subarachnoid blood. Neurosurgery. 1982;10(3):301-307.
11. Ibrahim GM, Vachhrajani S, Ilodigwe D, et al. Method of aneurysm treatment does not affect clot clearance after aneurysmal subarachnoid hemorrhage. Neurosurgery. 2012;70(1):102-109.

12. Angelos G Kolias, Jon Sen, Antonio Belli. Pathogenesis of Cerebral Vasospasm Following Aneurysmal Subarachnoid Hemorrhage: Putative Mechanisms and Novel Approaches. J Neurosci Res. 2009;87(1):1-11.

13. Josh D Bell, Shawn G Rhind, Alex P Di Battista, et al. Biomarkers of Glycocalyx Injury Are Associated With Delayed Cerebral Ischemia Following Aneurysmal Subarachnoid Hemorrhage: A Case Series Supporting a New Hypothesis. Neurocrit Care. 2017;26(3):339-347.

14. M Gazi Yasargill, Konstantinkasdaglis, Kewal K Jain, et al. Anatomical Observations of the subarachnoid cisterns of the brain during surgery. $J$ Neurosurg. 1976;44(3):298-302.

15. Roberto C Heros, Nicholas T Zervas, Vassilios Varsos. Cerebral Vasospasm after Subarachnoid Hemorrhage- An update. Ann Neurol. $1983 ; 14: 599-608$

16. Genya Odake, Ken Yoshikawa, Shoji Naruse. Vasospasm and adrenergic innervation of Circle of Willis. Japanese journal of surgery. 1974;4(4):222-230

17. Masataka Takahashi, Zhen-Du Zhang, R Loch Macdonald. Sphenopalatine Ganglion Stimulation for Vasospasm after Experimental Subarachnoid Hemorrhage. J Neurosurg. 2011;114(4):1104-1109. 